\title{
ESTIMATES OF CONVOLUTIONS OF CERTAIN NUMBER-THEORETIC ERROR TERMS
}

\section{ALEKSANDAR IVIĆ}

Received 13 May 2003

\begin{abstract}
Several estimates for the convolution function $C[f(x)]:=\int_{1}^{x} f(y) f(x / y)(d y / y)$ and its iterates are obtained when $f(x)$ is a suitable number-theoretic error term. We deal with the case of the asymptotic formula for $\int_{0}^{T}|\zeta(1 / 2+i t)|^{2 k} d t(k=1,2)$, the general Dirichlet divisor problem, the problem of nonisomorphic Abelian groups of given order, and the Rankin-Selberg convolution.
\end{abstract}

2000 Mathematics Subject Classification: 11N37, 11M06, 44A15.

1. Convolution functions and Mellin transforms. Let $\mathscr{L}$ denote the set of functions $f(x) \in L^{1}(1, \infty)$ for which there exists a constant $\alpha_{f} \geqslant 0$ such that

$$
f(x) \ll_{\varepsilon} x^{\alpha_{f}+\varepsilon} .
$$

Actually it is more precise to define $\alpha_{f}$ as the infimum of the constants for which (1.1) holds. Here and later $\varepsilon>0$ denotes arbitrarily small constants, not necessarily the same ones at each occurrence. The notation $A \ll_{\varepsilon} B$ (the same as $A=O_{\varepsilon}(B)$ ) means that $|A| \leqslant C(\varepsilon) B$ for some positive constant $C(\varepsilon)$, which depends only on $\varepsilon$. We define the convolution of functions $f, g \in \mathscr{L}$ as

$$
(f \odot g)(x):=\int_{1}^{x} f(y) g\left(\frac{x}{y}\right) \frac{d y}{y} .
$$

The operation $\odot$ is commutative, associative, and distributive with respect to ordinary addition. Note that $f \odot g \in \mathscr{L}$ since it obviously lies in $L^{1}(1, \infty)$ and, moreover,

$$
\int_{1}^{x} f(y) g\left(\frac{x}{y}\right) \frac{d y}{y} \ll_{\varepsilon} \int_{1}^{x} y^{\alpha_{f}+\varepsilon}\left(\frac{x}{y}\right)^{\alpha_{g}+\varepsilon} \frac{d y}{y} \ll_{\varepsilon} x^{\gamma+\varepsilon}
$$

with $\gamma=\gamma(f, g)=\max \left(\alpha_{f}, \alpha_{g}\right)$. We also set, if $f \in \mathscr{L}$,

$$
C[f(x)]:=(f \odot f)(x)=\int_{1}^{x} f(y) f\left(\frac{x}{y}\right) \frac{d y}{y} \quad(x \geqslant 1) .
$$

More generally, the iterates of $C[f(x)]$ are defined as

$$
C_{1}[f(x)] \equiv C[f(x)], \quad C_{k}[f(x)]:=C\left[C_{k-1}[f(x)]\right] \quad(x \geqslant 1, k \geqslant 2) .
$$


Obviously we have, in view of (1.1),

$$
C_{k}[f(x)] \ll_{\varepsilon, k} x^{\alpha_{f}+\varepsilon} .
$$

The main aim of this paper is to improve on (1.6) (i.e., obtain (1.6) with smaller exponent than $\alpha_{f}$ ) in case $f(x)$ represents a well-known number-theoretic error term. In Section 2, we will investigate the case of mean square or biquadrate of $|\zeta(1 / 2+i t)|$ and the error terms in the corresponding asymptotic formulas. In Section 4, we will deal with $\Delta_{k}(x)$, the error term in the (generalized) Dirichlet divisor problem (or the Piltz divisor problem, as it is also commonly called). The method of the proof clearly can be used to deal also with various other error terms in divisor problems (see, e.g., [5, Chapter 14]). We will treat in detail two such problems: nonisomorphic Abelian groups will be treated in Section 6. In Section 8, we will deal with the Rankin-Selberg convolution of holomorphic cusp forms. In all these cases the bound (1.6) will be improved.

The convolution functions $(f \odot g)(x)$ appear naturally in the following context. Let, henceforth, $s=\sigma+$ it denote a complex variable and let

$$
F^{*}(s) \equiv m[f(x)]:=\int_{1}^{\infty} f(x) x^{-s} d x
$$

denote the modified Mellin transform of $f(x) \in \mathscr{L}$. Then the integral in (1.7) converges absolutely at least for $\sigma>1+\alpha_{f}$, and in many cases one can obtain the analytic continuation of $F^{*}(s)$ to a region lying to the left of the line $\mathfrak{R e} s=1+\alpha_{f}$. We remark that the functions $F^{*}(s)$ arise naturally in the theory of the Riemann zeta-function $\zeta(s)$; in particular, in the study of its power moments (see, e.g., [15, 17]). The author noted in [15] that if $x^{-\sigma} f(x) \in L^{1}(1, \infty)$ and $f(x)$ is continuous for $x>1$, then the inversion formula

$$
f(x)=\frac{1}{2 \pi i} \int_{(\sigma)} F^{*}(s) x^{s-1} d s
$$

certainly holds, where $\int_{(\sigma)}$ denotes the integral over the line with real part $\sigma$. This is a consequence of properties of the standard Mellin transform $M[f(x)]=\int_{0}^{\infty} f(x) x^{s-1} d x$. The connection between $f \odot g$ and modified Mellin transforms is given in the following lemma.

LEMMA 1.1. Let $f, g \in \mathscr{L}$. Then

$$
m[(f \odot g)(x)]=m[f(x)] m[g(x)] .
$$

The functions $m\left[|\zeta(1 / 2+i x)|^{\ell}\right]$, when $\ell=2,4$, have been recently extensively investigated (see [14, 15, 17, 21, 24, 25]). Many results have been obtained on these fundamental objects pertaining to moments of $|\zeta(1 / 2+i x)|^{\ell}$ when $\ell=2,4$. In view of (1.9) it transpires that the convolutions $(f \odot g)(x)$ are important objects which, in particular, can shed light on moments of $|\zeta(1 / 2+i x)|$.

Proof of THE LEMma. We write the right-hand side of (1.9) as the double integral

$$
\int_{1}^{\infty} \int_{1}^{\infty} f(x) g(y)(x y)^{-s} d x d y,
$$


and make the change of variables $x=X / Y$ and $y=Y$. The Jacobian of the transformation equals $1 / Y$, and in view of (1.3) we obtain

$$
\begin{aligned}
F^{*}(s) G^{*}(s) & =m[f(x)] m[g(x)]=\int_{1}^{\infty} X^{-s} \int_{1}^{X} f\left(\frac{X}{Y}\right) g(Y) \frac{d Y}{Y} d X \\
& =\int_{1}^{\infty}(f \odot g)(x) x^{-s} d x \quad\left(\sigma>1+\max \left(\alpha_{f}, \alpha_{g}\right)\right) .
\end{aligned}
$$

It may be remarked that the Mellin transform $m[f(x)]$ is a continuous analogue of the zeta-function (Dirichlet series)

$$
F(s) \equiv \mathscr{F}[f(n)]:=\sum_{n=1}^{\infty} f(n) n^{-s},
$$

where $f(n)$ is a sequence (arithmetic function) such that $F(s)$ converges absolutely for $\sigma>\sigma_{f}$. In the above notation the analogue of (1.9) reads as

$$
\mathscr{F}[(f * g)(n)]=\mathscr{F}[f(n)] \mathscr{F}[g(n)],
$$

where

$$
(f * g)(n):=\sum_{d \mid n} f(d) g\left(\frac{n}{d}\right)
$$

is the ordinary convolution of the sequences (arithmetic functions) $f(n)$ and $g(n)$. Hence in this analogy the convolution functions $(f \odot g)(x)$ and $(f * g)(n)$ correspond to one another.

We now turn to the functions $C[f(x)]$. If we set $f \equiv g$ in (1.9) and use the inversion formula (1.8), we obtain that

$$
C[f(x)]=\frac{1}{2 \pi i} \int_{(\sigma)}\left(F^{*}(s)\right)^{2} x^{s-1} d s
$$

for a suitable $\sigma$. Thus, relation (1.15) furnishes the tool to estimate $C[f(x)]$ provided that we have a good mean-square estimate for $F^{*}(s)$. More generally, by iteration the lemma yields

$$
\left(F^{*}(s)\right)^{2^{k}}=\int_{1}^{\infty} C_{k}[f(x)] x^{-s} d x \quad\left(\sigma \geqslant \sigma_{0, k}\right),
$$

and from (1.8) and (1.15) we further have, for a suitable $\sigma_{1, k}$,

$$
C_{k}[f(x)]=\frac{1}{2 \pi i} \int_{\left(\sigma_{1, k}\right)}\left(F^{*}(s)\right)^{2^{k}} x^{s-1} d s .
$$


Now we will use the foregoing discussion and formulate a general result on the estimation of $C[f(x)]$. To do this, suppose that the sequence $\left\{\ell_{n}\right\}_{n=1}^{\infty}$ satisfies $1=\ell_{1}<$ $\ell_{2}<\cdots, \lim _{n \rightarrow \infty} \ell_{n}=+\infty$, and let

$$
\mathscr{A}(s):=\sum_{n=1}^{\infty} a_{n} \ell_{n}^{-s} \quad\left(\sigma>\sigma_{0}\right)
$$

be a Dirichlet series such that the summatory function

$$
A(x):=\sum_{n \leqslant x} a_{n}
$$

may be written in the form

$$
A(x):=\sum_{i=1}^{k} \sum_{j=0}^{M_{i}} c_{i, j} x^{\alpha_{j}} \log ^{j} x+u(x) .
$$

Here the $c_{i, j}$ 's are real constants with $c_{1, M_{1}}>0$ and $\alpha_{1}>\alpha_{2}>\cdots>\alpha_{k}>0$. The condition $\ell_{1}=1$ is technically convenient and can be always attained if, instead of $\mathscr{A}(s)$, one considers $\mathscr{A}(s)$ multiplied by a suitable constant. The Laurent coefficients of such functions, which frequently occur in analytic number theory, were investigated by the author in [10]. The function $u(x)$ is to be considered as the error term in the asymptotic formula for $A(x)$. For this to hold, it is enough to assume that the mean-square estimate

$$
\int_{0}^{X} u^{2}(x) d x \ll X^{1+2 \beta} \quad\left(0<\beta<\alpha_{k}\right)
$$

holds, which is fairly easy to obtain in practice. Our aim is to derive an upper bound for $C[u(x)]$, contained in the following theorem.

THEOREM 1.2. Let the above hypotheses on $A(x)$ and $u(x)$ hold. Furthermore suppose that $\mathscr{A}(s)$ admits analytic continuation to $\mathfrak{R e} s>0$, where it is regular except for the poles at $s=\alpha_{1}, \alpha_{2}, \ldots, \alpha_{k}$ which are of order $M_{1}+1, M_{2}+1, \ldots, M_{k}+1$, respectively. If

$$
\int_{T}^{2 T}\left|\mathscr{A}\left(\sigma_{1}+i t\right)\right|^{2} d t \ll T^{2-\delta}
$$

holds for some $\delta>0$ and $0<\sigma_{1}<\alpha_{k}$, then

$$
C[u(x)]=\int_{1}^{x} u(y) u\left(\frac{x}{y}\right) \frac{d y}{y} \ll x^{\sigma_{1}}
$$

Proof of TheOrem 1.2. We have, for $\sigma>\alpha$ and $j=0,1,2, \ldots$,

$$
\int_{1}^{\infty} x^{\alpha-s-1} \log ^{j} x \cdot d x=\int_{0}^{\infty} e^{-(s-\alpha) u} u^{j} d u=(s-\alpha)^{-j-1} \int_{0}^{\infty} e^{-t} t^{j} d t=j !(s-\alpha)^{-j-1} .
$$


Hence by the Stieltjes integral representation and (1.18), (1.19), and (1.20) it follows that

$$
\begin{aligned}
\mathscr{A}(s)= & \int_{1-0}^{\infty} x^{-s} d A(x) \\
= & \sum_{i=1}^{k} \sum_{j=0}^{M_{i}} c_{i, j} \int_{1}^{\infty}\left(\alpha_{i} x^{\alpha_{i}-1} \log ^{j} x+j x^{\alpha_{i}-1} \log ^{j-1} x\right) x^{-s} d x \\
& +\int_{1-0}^{\infty} x^{-s} d u(x) \\
= & \sum_{i=1}^{k}\left(\sum_{j=1}^{M_{i}} c_{i, j}\left(\alpha_{i}+1\right) j !\left(s-\alpha_{i}\right)^{-j-1}+c_{i, 0} \alpha_{i}\left(s-\alpha_{i}\right)^{-1}\right) \\
& -u(1-0)+s \int_{1}^{\infty} u(x) x^{-s-1} d x
\end{aligned}
$$

In view of (1.21) and the Cauchy-Schwarz inequality for integrals, it follows that the last integral above converges absolutely at least for $\sigma>\beta$. Therefore the function

$$
\mathscr{B}(s):=\mathscr{A}(s)-\sum_{i=1}^{k}\left(\sum_{j=1}^{M_{i}} c_{i, j}\left(\alpha_{i}+1\right) j !\left(s-\alpha_{i}\right)^{-j-1}+c_{i, 0} \alpha_{i}\left(s-\alpha_{i}\right)^{-1}\right)+u(1-0)
$$

is regular for $\sigma>\beta$. Consequently, by (1.8) and (1.9) we have

$$
C[u(x)]=\frac{1}{2 \pi i} \int_{\left(\sigma_{2}\right)} \mathscr{B}^{2}(s) s^{-2} x^{s} d s
$$

for some suitable $\sigma_{2}>0$. This is because we have

$$
C\left[\frac{u(x)}{x}\right]=\frac{1}{x} \int_{1}^{x} u(y) u\left(\frac{x}{y}\right) \frac{d y}{y}=\frac{1}{x} C[u(x)] .
$$

Note that

$$
|\mathscr{B}(\sigma+i t)|^{2} \ll|\mathscr{A}(\sigma+i t)|^{2}+1 \quad(|t| \geqslant 1)
$$

if (1.26) holds, which means that if we use (1.21), then we may shift the line of integration in (1.27) to $\mathfrak{R e} s=\sigma_{1}$. Moreover, the ensuing integral will converge absolutely, and we obtain

$$
C[u(x)]=\frac{1}{2 \pi i} \int_{\left(\sigma_{1}\right)} \mathscr{B}^{2}(s) s^{-2} x^{s} d s \ll x^{\sigma_{1}}
$$

as asserted.

2. The case of $|\zeta(1 / 2+i t)|^{2}$ and $|\zeta(1 / 2+i t)|^{4}$. We will formulate in this section our results for the convolutions of $|\zeta(1 / 2+i t)|^{2}$ and $|\zeta(1 / 2+i t)|^{4}$, and postpone the proofs to Section 3. We will also deal with the error terms in the corresponding asymptotic formulas for the mean values of $|\zeta(1 / 2+i t)|^{2}$ and $|\zeta(1 / 2+i t)|^{4}$. Namely, mean values of $|\zeta(1 / 2+i t)|$ are fundamental in the theory and applications of $\zeta(s)$ 
(see the author's monographs [5, 9] for an extensive account). For $k \geqslant 1$ a fixed integer, let

$$
\int_{0}^{T}\left|\zeta\left(\frac{1}{2}+i t\right)\right|^{2 k} d t=T P_{k^{2}}(\log T)+E_{k}(T)
$$

where for some suitable coefficients $a_{j, k}$ one has

$$
P_{k^{2}}(y)=\sum_{j=0}^{k^{2}} a_{j, k} y^{j}
$$

In particular,

$$
P_{1}(y)=y+2 \gamma-1-\log (2 \pi)
$$

where $\gamma=-\Gamma^{\prime}(1)=0.5772157 \ldots$ is Euler's constant. One hopes that

$$
E_{k}(T)=o(T) \quad(T \longrightarrow \infty)
$$

will hold for every fixed integer $k \geqslant 1$, but so far this is known to be true only in the cases $k=1$ and $k=2$, when $E_{k}(T)$ is a true error term in (2.1). The best unconditional bound for $E(T) \equiv E_{1}(T)$ is due to Huxley [4]. This is

$$
E_{1}(T) \ll_{\varepsilon} T^{137 / 432+\varepsilon}, \quad \frac{137}{432}=0.31713 \ldots
$$

We have $\Omega$-results in the case $k=1,2$, which show that $E_{1}(T)$ and $E_{2}(T)$ cannot always be small. Hafner and the author [2] proved that

$$
\begin{aligned}
& E_{1}(T)=\Omega_{+}\left((T \log T)^{1 / 4}(\log \log T)^{(3+\log 4) / 4} e^{-C \sqrt{\log \log \log T}}\right), \\
& E_{1}(T)=\Omega_{-}\left(T^{1 / 4} \exp \left(\frac{D(\log \log T)^{1 / 4}}{(\log \log \log T)^{3 / 4}}\right)\right)
\end{aligned}
$$

for some absolute constants $C, D>0$. As usual, the notation $f=\Omega_{+}(g)$ means that $\limsup f / g>0, f=\Omega_{-}(g)$ means that liminf $f / g<0$, and $f=\Omega_{ \pm}(g)$ means that both $f=\Omega_{+}(g)$ and $f=\Omega_{-}(g)$ hold. These $\Omega$-results support the conjecture that $E_{1}(T) \ll_{\varepsilon}$ $T^{1 / 4+\varepsilon}$, which is also supported by the mean-square formula (see [9, Theorem 2.4])

$$
\int_{0}^{T} E_{1}^{2}(t) d t=c T^{3 / 2}+O\left(T \log ^{5} T\right), \quad c=\frac{2}{3}(2 \pi)^{-1 / 2} \frac{\zeta^{4}(3 / 2)}{\zeta(3)}=10.3047 \ldots
$$

In case $k=2,(2.1)$ becomes

$$
\int_{0}^{T}\left|\zeta\left(\frac{1}{2}+i t\right)\right|^{4} d t=T P_{4}(\log T)+E_{2}(T), \quad P_{4}(x)=\sum_{j=0}^{4} a_{j} x^{j}
$$


where $a_{4}=1 /\left(2 \pi^{2}\right)$ and for the evaluation of the remaining $a_{j}$ 's see [11]. It is known (see $[9,12,14,19,23,24,25])$ that

$$
E_{2}(T)=O\left(T^{2 / 3} \log ^{8} T\right), \quad E_{2}(T)=\Omega_{ \pm}\left(T^{1 / 2}\right),
$$

and that (see $[13,18,25]$ for the lower bound)

$$
\int_{0}^{T} E_{2}^{2}(t) d t \ll T^{2} \log ^{22} T, \quad \int_{0}^{T} E_{2}^{2}(t) d t \gg T^{2} .
$$

The bounds in (2.10) support the conjecture that $E_{2}(T) \ll_{\varepsilon} T^{1 / 2+\varepsilon}$. Related to the functions $E_{k}(T)$ are the functions

$$
\mathscr{L}_{k}(s)=m\left[\left|\zeta\left(\frac{1}{2}+i x\right)\right|^{2 k}\right]=\int_{1}^{\infty}\left|\zeta\left(\frac{1}{2}+i x\right)\right|^{2 k} x^{-s} d x
$$

The most important cases are $k=1,2$, when the integral in (2.11) converges absolutely for $\sigma>1$, and when one can obtain analytic continuation to the left of the line $\mathfrak{R e} s=1$. Namely, for $\sigma>1$, we have from (2.1), with $Q_{1}(y)=P_{1}(y)+P_{1}^{\prime}(y)$,

$$
\begin{aligned}
\mathscr{L}_{1}(s) & =\int_{1}^{\infty}\left(Q_{1}(\log x)+E^{\prime}(x)\right) x^{-s} d x \\
& =\frac{1}{(s-1)^{2}}+\frac{2 \gamma-\log (2 \pi)}{s-1}-E(1)+s \int_{1}^{\infty} E(x) x^{-s-1} d x,
\end{aligned}
$$

and the last integral converges absolutely for $\sigma>1 / 4$ (by (2.7)), thereby providing the analytic continuation of $\mathscr{E}_{1}(s)$ to the region $\sigma>1 / 4$.

Similarly (by using the upper bound in (2.10)) we obtain, for $\sigma>1 / 2$,

$$
\mathscr{L}_{2}(s)=\sum_{j=0}^{5} c_{j}(s-1)^{-j}+s \int_{1}^{\infty} E_{2}(x) x^{-s-1} d x
$$

with suitable constants $c_{j}$.

Before we formulate our results on upper bounds for $C\left[|\zeta(1 / 2+i x)|^{2 k}\right](k=1,2)$, we note that it is fairly easy to obtain a lower bound. Namely, we have

$$
\begin{aligned}
C\left[\left|\zeta\left(\frac{1}{2}+i x\right)\right|^{2 k}\right] & \geqslant \int_{(1 / 2) x}^{x}\left|\zeta\left(\frac{1}{2}+i y\right)\right|^{2 k}\left|\zeta\left(\frac{1}{2}+i \frac{x}{y}\right)\right|^{2 k} \frac{d y}{y} \\
& \geqslant \min _{u \in[1,2]}\left|\zeta\left(\frac{1}{2}+i u\right)\right|^{2 k} \int_{(1 / 2) x}^{x}\left|\zeta\left(\frac{1}{2}+i y\right)\right|^{2 k} \frac{d y}{y} \\
& \gg(\log x)^{k^{2}+1},
\end{aligned}
$$

on using (2.1) in conjunction with (2.5) and (2.9). Our bounds are given by the following theorem. 
THEOREM 2.1. We have

$$
\begin{gathered}
C\left[\left|\zeta\left(\frac{1}{2}+i x\right)\right|^{2}\right] \ll_{\varepsilon} x^{\varepsilon}, \\
C\left[\left|\zeta\left(\frac{1}{2}+i x\right)\right|^{4}\right] \ll_{\varepsilon} x^{\varepsilon} \min \left(x^{4 \mu(1 / 2)}, x^{2 \mu(1 / 2)+1 / 4}, x^{1 / 3}\right),
\end{gathered}
$$

where, for given $\sigma$,

$$
\mu(\sigma)=\limsup _{t \rightarrow \infty} \frac{\log |\zeta(\sigma+i t)|}{\log t} .
$$

COROLLARY 2.2. If the Lindelöf hypothesis that $\zeta(1 / 2+i t) \ll_{\varepsilon}|t|^{\varepsilon}$ holds (i.e., $\mu(1 / 2)$ $=0)$, then

$$
C\left[\left|\zeta\left(\frac{1}{2}+i x\right)\right|^{4}\right] \ll_{\varepsilon} x^{\varepsilon} .
$$

The bounds (2.15) and (2.18) are, in view of (2.14), essentially the best possible, but it seems that (2.18) cannot be proved at present without assuming the (yet unproved) Lindelöf hypothesis. In view of the best known result $\mu(1 / 2) \leqslant 89 / 570$ (see Huxley [4]), it follows that (2.16) yields unconditionally the bound

$$
C\left[\left|\zeta\left(\frac{1}{2}+i x\right)\right|^{4}\right] \ll_{\varepsilon} x^{1 / 3+\varepsilon} .
$$

Our bounds for $C_{k}[E(x)]$, when $k=1,2$, and for $C\left[E_{2}(x)\right]$ are contained in the following theorem.

THEOREM 2.3. We have

$$
\begin{gathered}
C[E(T)] \ll_{\varepsilon} T^{1 / 4+\varepsilon}, \quad C_{2}[E(T)] \ll_{\varepsilon} T^{3 / 20+\varepsilon}, \\
C\left[E_{2}(T)\right] \ll_{\varepsilon} T^{1 / 2+\varepsilon} .
\end{gathered}
$$

The bounds in (2.20) and (2.21) are indeed better than the ones furnished by (1.6) in conjunction with (2.5) and the upper bound in (2.9).

3. Proofs of Theorems 2.1 and 2.3. We begin with the proof of Theorem 2.1, noting that trivially

$$
C\left[\left|\zeta\left(\frac{1}{2}+i x\right)\right|^{2 k}\right] \ll_{\varepsilon, k} x^{2 k \mu(1 / 2)+\varepsilon},
$$

by the definition of the function $\mu(\sigma)$. On the other hand, we have, in view of (2.8) $\left(Q_{4}(y)=P_{4}(y)+P_{4}^{\prime}(y)\right)$,

$$
C\left[\left|\zeta\left(\frac{1}{2}+i x\right)\right|^{4}\right]=2 H(x),
$$


with

$$
\begin{aligned}
H(x) & :=\int_{\sqrt{x}}^{x}\left|\zeta\left(\frac{1}{2}+i u\right)\right|^{4}\left|\zeta\left(\frac{1}{2}+i \frac{x}{u}\right)\right|^{4} \frac{d u}{u} \\
& =\int_{\sqrt{x}}^{x}\left(Q_{4}\left(\log \frac{x}{u}\right)+E_{2}^{\prime}\left(\frac{x}{u}\right)\right)\left(Q_{4}(\log u)+E_{2}^{\prime}(u)\right) \frac{d u}{u} \\
& =H_{1}(x)+H_{2}(x)+H_{3}(x)+H_{4}(x),
\end{aligned}
$$

say. Here, we set

$$
\begin{aligned}
H_{1}(x) & :=\int_{\sqrt{x}}^{x} Q_{4}\left(\log \frac{x}{u}\right) Q_{4}(\log u) \frac{d u}{u} \ll \log ^{9} x \\
H_{2}(x) & =H_{3}(x)=\int_{\sqrt{x}}^{x} Q_{4}\left(\log \frac{x}{u}\right) E_{2}^{\prime}(u) \frac{d u}{u} \\
& =\left.\frac{E_{2}(u)}{u} Q_{4}\left(\log \frac{x}{u}\right)\right|_{\sqrt{x}} ^{x}+\int_{\sqrt{x}}^{x} \frac{E_{2}(u)}{u^{2}}\left(Q_{4}\left(\log \frac{x}{u}\right)-Q_{4}^{\prime}\left(\log \frac{x}{u}\right)\right) d u \\
& \ll 1
\end{aligned}
$$

in view of (2.9). The main problem is the estimation of the function

$$
H_{4}(x):=\int_{\sqrt{x}}^{x} E_{2}^{\prime}(u) E_{2}^{\prime}\left(\frac{x}{u}\right) \frac{d u}{u} .
$$

Integration by parts gives

$$
H_{4}(x):=\left.E_{2}(u) E_{2}^{\prime}\left(\frac{x}{u}\right) \frac{1}{u}\right|_{\sqrt{x}} ^{x}+\int_{\sqrt{x}}^{x} E_{2}(u)\left(\frac{1}{u^{2}} E_{2}^{\prime}\left(\frac{x}{u}\right)+\frac{x}{u^{3}} E_{2}^{\prime \prime}\left(\frac{x}{u}\right)\right) d u .
$$

Since one has, by differentiating (2.8),

$$
E_{2}^{\prime}(y) \ll_{\varepsilon} y^{4 \mu(1 / 2)+\varepsilon}, \quad E_{2}^{\prime \prime}(y) \ll_{\varepsilon} y^{4 \mu(1 / 2)+\varepsilon},
$$

this yields

$$
\begin{aligned}
H_{4}(x) & \ll_{\varepsilon} 1+x^{1 / 3+\varepsilon} x^{-1 / 2} x^{2 \mu(1 / 2)+\varepsilon}+x \int_{\sqrt{x}}^{x}\left|E_{2}(u)\right| u^{-3}\left(\frac{x}{u}\right)^{4 \mu(1 / 2)+\varepsilon} d u \\
& \ll_{\varepsilon} 1+x^{2 \mu(1 / 2)-1 / 6+\varepsilon}+x^{2 \mu(1 / 2)+1 / 4+(1 / 2) \varepsilon} \\
& \ll_{\varepsilon} x^{2 \mu(1 / 2)+1 / 4+\varepsilon},
\end{aligned}
$$

where we used the Cauchy-Schwarz inequality for integrals and (2.10). This proves the second part of the bound on the right-hand side of (2.16). To obtain the third part, note that by [15, equation (3.23)] we have

$$
\begin{aligned}
\int_{T}^{2 T} & \left|\mathscr{L}_{2}(\sigma+i t)\right|^{2} d t \\
& \ll_{\varepsilon} T^{-1}+T^{\varepsilon}\left(T^{2 c-2 \sigma} \int_{T}^{2 T}\left|\mathscr{L}_{2}(c+i t)\right|^{2} d t+T^{2-2 \sigma}+T^{5-6 \sigma}\right)
\end{aligned}
$$


for $1 / 2<c \leqslant \sigma, T \geqslant 3$. Since (see [17])

$$
\int_{0}^{T}\left|\mathscr{L}_{2}(\sigma+i t)\right|^{2} d t \ll T^{(1 / 3)(10-8 \sigma)} \log ^{C} T \quad\left(\frac{1}{2}<\sigma<1\right)
$$

holds, this gives

$$
\int_{T}^{2 T}\left|\mathscr{E}_{2}(\sigma+i t)\right|^{2} d t \ll_{\varepsilon} T^{-1}+T^{8 / 3-2 \sigma+\varepsilon} \quad\left(\sigma \geqslant \frac{5}{6}\right) .
$$

Analyzing the proof of the above bound in [15], it transpires that if we use $\mathscr{L}_{1}(s)$ instead of $\mathscr{L}_{2}(s)$, then in [15, equation (3.25)] instead of the term $T^{2} X^{1-2 \sigma} \log ^{C} X$ there will appear (in view of (2.7))

$$
T^{2} X^{1 / 2-2 \sigma} \log ^{C} X
$$

The optimal choice for $X$ will now satisfy

$$
T^{-1} X^{2-2 \sigma}=T^{2} X^{1 / 2-2 \sigma},
$$

giving $X=T^{2}$. It follows that, in the notation of [15],

$$
\int_{T}^{2 T}\left(\left|I_{1}(s)\right|^{2}+\left|I_{2}(s)\right|^{2}\right) d t \ll_{\varepsilon} T^{-1}+T^{3-4 \sigma} \log ^{C} T
$$

therefore one obtains

$$
\begin{aligned}
\int_{T}^{2 T} & \left|\mathscr{L}_{1}(\sigma+i t)\right|^{2} d t \\
& \ll_{\varepsilon} T^{-1}+T^{\varepsilon}\left(T^{2 c-2 \sigma} \int_{T}^{2 T}\left|\mathscr{L}_{1}(c+i t)\right|^{2} d t+T^{2-2 \sigma}+T^{3-4 \sigma}\right)
\end{aligned}
$$

for $1 / 2<c \leqslant \sigma, T \geqslant 3$. Since $3-4 \sigma \leqslant 2-2 \sigma$ for $\sigma \geqslant 1 / 2$ and we have (see [17])

$$
\int_{1}^{T}\left|\mathscr{L}_{1}(\sigma+i t)\right|^{2} d t \ll_{\varepsilon} T^{2-2 \sigma+\varepsilon} \quad\left(\frac{1}{2} \leqslant \sigma \leqslant 1\right),
$$

it follows that

$$
\int_{T}^{2 T}\left|\mathscr{L}_{1}(\sigma+i t)\right|^{2} d t \ll_{\varepsilon} T^{-1}+T^{2-2 \sigma+\varepsilon} \quad\left(\sigma \geqslant \frac{1}{2}\right)
$$

By (2.11), (1.9), and the inversion formula (1.8), we have

$$
C\left[\left|\zeta\left(\frac{1}{2}+i x\right)\right|^{2 k}\right]=\frac{1}{2 \pi i} \int_{(\sigma(k))} \mathscr{E}_{k}^{2}(s) x^{s-1} d s
$$

for sufficiently large $\sigma(k)(>1)$. In view of the mean-square bounds for $k=1$ and $k=2$, given by (3.17) and (3.11), respectively, we may in (3.18) take $\sigma(1)=1+\varepsilon$ and $\sigma(2)=4 / 3+\varepsilon$. This yields (2.15) and the last bound in (2.16). 
We pass now to the proof of Theorem 2.3. Let

$$
z_{1}(s):=\mathscr{L}_{1}(s)-\frac{1}{(s-1)^{2}}+\frac{\log (2 \pi)-2 \gamma}{s-1}+E(1) .
$$

Then, by (2.12), $z_{1}(s)$ is regular for $\sigma>1 / 4$ and we have

$$
z_{1}(s)=s \int_{1}^{\infty} \frac{E(x)}{x} \cdot x^{-s} d x \quad\left(\sigma>\frac{1}{4}\right) .
$$

Therefore, (1.8) and (1.9) give

$$
C\left[\frac{E(x)}{x}\right]=\frac{1}{2 \pi i} \int_{(1)} \frac{z_{1}^{2}(s)}{s^{2}} x^{s-1} d s .
$$

Note that, by using (1.28), it follows that

$$
C[E(x)]=\frac{1}{2 \pi i} \int_{(1)} \frac{z_{1}^{2}(s)}{s^{2}} x^{s} d s .
$$

But $\mathscr{L}_{1}(s)$ admits analytic continuation to the region $\sigma>-1 / 4$ (see [17]). Moreover, we have

$$
\int_{1}^{T}\left|\mathscr{L}_{1}(\sigma+i t)\right|^{2} d t \ll_{\varepsilon} T^{3-4 \sigma+\varepsilon} \quad\left(0 \leq \sigma \leq \frac{1}{2}\right)
$$

and (see Jutila [21])

$$
\mathscr{Z}_{1}(\sigma+i t) \ll_{\varepsilon}|t|^{(1 / 3)(3-4 \sigma)+\varepsilon} \quad\left(0 \leq \sigma \leq \frac{1}{2}\right) .
$$

From (3.19) and (3.23), it follows that we may shift the line of integration in (3.22) to $\mathfrak{R e} s=1 / 4+\varepsilon$ and deduce that the first bound in (2.20) holds. To derive the second bound note that (1.8) and (3.20) yield

$$
z_{1}^{4}(s)=s^{4}\left(\int_{1}^{\infty} C[E(x)] x^{-s-1} d x\right)^{2}=s^{4} \int_{1}^{\infty} C_{2}[E(x)] x^{-s-1} d x \quad\left(\sigma>\frac{1}{4}\right) .
$$

Therefore,

$$
C_{2}[E(x)]=\frac{1}{2 \pi i} \int_{(1)} \frac{z_{1}^{4}(s)}{s^{4}} x^{s} d s
$$

Writing $z_{1}^{4}=z_{1}^{2} \cdot z_{1}^{2}$ and using (3.23) and (3.24), we obtain

$$
\int_{T}^{2 T}\left|z_{1}(\sigma+i t)\right|^{4} t^{-4} d t \ll_{\varepsilon} T^{3-4 \sigma+(2 / 3)(3-4 \sigma)-4+\varepsilon} \ll_{\varepsilon} T^{-\varepsilon} \quad\left(0<\sigma<\frac{1}{2}\right)
$$

for $\sigma>3 / 20$ and sufficiently small $\varepsilon$. It follows that

$$
\int_{(3 / 20+\varepsilon)} z_{1}^{4}(s) s^{-4} x^{s} d s \ll_{\varepsilon} x^{3 / 20+\varepsilon},
$$


proving the second upper bound in (2.20). Bounds for $C_{2^{k}}[E(x)]$ may be obtained in a similar fashion.

To obtain (2.21), we set (cf. (2.13))

$$
z_{2}(s)=\mathscr{L}_{2}(s)-\sum_{j=0}^{5} c_{j}(s-1)^{-j},
$$

so that $z_{2}(s)$ is regular for $\sigma>1 / 2$. Then, analogously to (3.22), we have

$$
C\left[E_{2}(x)\right]=\frac{1}{2 \pi i} \int_{(1)} \frac{z_{2}^{2}(s)}{s^{2}} x^{s} d s .
$$

The proof is completed if we move the line of integration in (3.30) to $\mathfrak{R e} s=1 / 2+\varepsilon$ and use the mean-square estimate (see [17])

$$
\int_{0}^{T}\left|\mathscr{L}_{2}(\sigma+i t)\right|^{2} d t \ll T^{(10-8 \sigma) / 3} \log ^{C} T \quad\left(\frac{1}{2}<\sigma<1\right)
$$

and the fact that

$$
\left|z_{2}(\sigma+i t)\right|^{2} \ll\left|\mathscr{L}_{2}(\sigma+i t)\right|^{2}+1 \quad\left(\sigma>\frac{1}{2},|t| \geqslant 1\right) .
$$

We note that Motohashi [24] established that $\mathscr{L}_{2}(s)$ has infinitely many poles on the line $\mathfrak{R e} s=1 / 2$, so that by this method one cannot improve the bound

$$
C_{2}\left[E_{2}(x)\right] \ll_{\varepsilon} x^{1 / 2+\varepsilon},
$$

which follows from (2.21) by trivial estimation.

4. The Dirichlet divisor problem. The (generalized) Dirichlet divisor problem, or the Piltz divisor problem, as it is also sometimes called, consists of the estimation of the function

$$
\Delta_{k}(x):=\sum_{n \leqslant x} d_{k}(n)-x p_{k-1}(\log x)
$$

The arithmetic function $d_{k}(n)$ denotes the number of ways $n$ can be written as a product of $k$ factors $\left(d_{2}(n) \equiv d(n)\right.$ is the number of divisors of $n$ ), and $p_{k-1}(x)$ is a suitable polynomial in $x$ of degree $k-1$. It may be evaluated explicitly (see the monographs $[5,30]$ for an extensive account on divisor problems) as

$$
p_{k-1}(\log x)=\operatorname{Res}_{s=1} \frac{x^{s-1}}{s} \zeta^{k}(s),
$$

and in particular $p_{1}(y)=y+2 \gamma-1$ since

$$
\sum_{n=1}^{\infty} d_{k}(n) n^{-s}=\zeta^{k}(s) \quad(\sigma>1, k \in \mathbb{N}) .
$$


In connection with $\Delta_{k}(x)$, one defines the constants

$$
\alpha_{k}=\inf \left\{a: \Delta_{k}(x) \ll x^{a}\right\}, \quad \beta_{k}=\inf \left\{b: \int_{0}^{x} \Delta_{k}^{2}(y) d y \ll x^{1+2 b}\right\} .
$$

For a discussion about estimates of $\alpha_{k}$ and $\beta_{k}$, the reader is referred to the paper of Ouellet and the author [20].

Our results on $C_{k}[\Delta(x)]$ and $C\left[\Delta_{k}(x)\right]$ are contained in the following theorem.

THEOREM 4.1. For $k \geqslant 1$,

$$
C_{k}[\Delta(x)] \ll_{\varepsilon, k} x^{2^{-k-1}+\varepsilon} .
$$

Also,

$$
\begin{aligned}
& C\left[\Delta_{3}(x)\right] \ll_{\varepsilon} x^{1 / 3+\varepsilon}, \quad C\left[\Delta_{4}(x)\right] \ll_{\varepsilon} x^{3 / 8+\varepsilon}, \\
& C\left[\Delta_{5}(x)\right] \ll_{\varepsilon} x^{9 / 20+\varepsilon}, \quad C\left[\Delta_{6}(x)\right] \ll_{\varepsilon} x^{1 / 2+\varepsilon} .
\end{aligned}
$$

5. Proof of Theorem 4.1. From (4.1) and (4.3) one has, for $\sigma>1$ and $q_{k-1}(y)=$ $p_{k-1}(y)+p_{k-1}^{\prime}(y)$,

$$
\begin{aligned}
\zeta^{k}(s) & =\int_{1-0}^{\infty} x^{-s} d\left(\sum_{n \leqslant x} d_{k}(n)\right) \\
& =\int_{1}^{\infty} x^{-s} q_{k-1}(\log x) d x+\int_{1-0}^{\infty} x^{-s} d \Delta_{k}(x) \\
& =\sum_{j=0}^{k} \frac{b_{j, k}}{(s-1)^{j}}+s \int_{1}^{\infty} \Delta_{k}(x) x^{-s-1} d x
\end{aligned}
$$

with suitable constants $b_{j, k}$. By using the Cauchy-Schwarz inequality for integrals and (4.4), it is seen that the last integral in (5.1) converges for $\sigma>\beta_{k}$. Consequently, the function

$$
Z_{k}(s):=\zeta^{k}(s)-\sum_{j=0}^{k} \frac{b_{j, k}}{(s-1)^{j}}
$$

is regular for $\sigma>\beta_{k}$. To prove (4.5), note that from (5.1) and (5.2) we obtain

$$
Z_{2}^{2}(s)=s^{2} \int_{1}^{\infty} C[\Delta(x)] x^{-s-1} d x
$$

and, more generally,

$$
Z_{2}^{2^{k}}(s)=s^{2^{k}} \int_{1}^{\infty} C_{k}[\Delta(x)] x^{-s-1} d x \quad(k \geqslant 1),
$$

which is valid at least for $\sigma>1 / 3$, in view of the classical bound $\Delta(x) \ll x^{1 / 3}$. From (5.4) we obtain, analogously to (3.26),

$$
C_{k}[\Delta(x)]=\frac{1}{2 \pi i} \int_{(1)} Z_{2}^{2^{k}}(s) s^{-2^{k}} x^{s} d s \quad(k \geqslant 1) .
$$


Now we invoke the functional equation (see, e.g., [5, Chapter 1]) for $\zeta(s)$, namely,

$$
\zeta(s)=\chi(s) \zeta(1-s), \quad \chi(s)=2^{s} \pi^{s-1} \sin \left(\frac{1}{2} \pi s\right) \Gamma(1-s),
$$

with

$$
\chi(s)=\left(\frac{2 \pi}{t}\right)^{\sigma+i t-1 / 2} e^{i(t+(1 / 4) \pi)} \cdot\left(1+O\left(\frac{1}{t}\right)\right) \quad(t \geqslant 2) .
$$

This gives, for $0<\sigma<1 / 2$,

$$
\int_{T}^{2 T}\left|Z_{2}(\sigma+i t)\right|^{2^{k}} t^{-2^{k}} d t \ll T^{-\sigma 2^{k+1}} \int_{T}^{2 T}|\zeta(1-\sigma+i t)|^{2^{k+1}} d t+T^{1-2^{k}}
$$

We choose

$$
\sigma=\frac{1}{2^{k+1}}+\varepsilon
$$

and note that

$$
\int_{T}^{2 T}\left|\zeta\left(1-2^{-m}+i t\right)\right|^{2^{m}} d t \ll_{\varepsilon, m} T^{1+\varepsilon} \quad(m \geqslant 1) .
$$

This follows from the bounds in [5, Chapter 8], in particular from [5, equation (8.102)] for $m \geqslant 4$. Since $1-\sigma 2^{k+1}<0$ for $\sigma=1 / 2^{k+1}+\varepsilon$, it follows from (5.8) that we may shift the line of integration in (5.5) to $\mathfrak{R e} s=2^{-k-1}+\varepsilon$ and obtain (4.5).

The proofs of the bounds in (4.6) are in a similar vein, and may be derived by using Theorem 1.2. We will deal only with the cases $k=5$ and $k=6$. In the former case we have, analogously to (5.5),

$$
C\left[\Delta_{5}(x)\right]=\frac{1}{2 \pi i} \int_{(1)} Z_{5}(s) s^{-2} x^{s} d s .
$$

By the functional equation for $\zeta(s)$ and (see Zhang [31] and also Heath-Brown [3])

$$
\int_{0}^{T}\left|\zeta\left(\frac{11}{20}+i t\right)\right|^{10} d t \ll T^{3 / 2} \log ^{50} T
$$

we obtain, for arbitrarily small $\delta>0$,

$$
\int_{T}^{2 T}|\zeta(\sigma+i t)|^{10} t^{-2} d t \ll T^{3-10 \sigma} \int_{T}^{2 T}|\zeta(1-\sigma+i t)|^{10} d t \ll T^{-\delta}
$$

for $\sigma=9 / 20+\varepsilon$ and sufficiently small $\varepsilon=\varepsilon(\delta)$. Thus (1.22) of Theorem 1.2 holds with $\sigma_{1}=9 / 20+\varepsilon$, implying $C\left[\Delta_{5}(x)\right] \ll_{\varepsilon} x^{9 / 20+\varepsilon}$. The bound $C\left[\Delta_{6}(x)\right] \ll_{\varepsilon} x^{1 / 2+\varepsilon}$ follows analogously from

$$
\int_{0}^{T}\left|\zeta\left(\frac{1}{2}+i t\right)\right|^{12} d t \ll T^{2} \log ^{17} T
$$

We note that the exponents in (4.6) correspond to the best known values (cf. [5, 20]) $\beta_{3}=1 / 3, \beta_{4}=3 / 8, \beta_{5} \leqslant 9 / 20$ (see [31]), and $\beta_{6} \leqslant 1 / 2$, since in both cases the exponents 
depend on similar mean-square estimates (cf. (1.22)). Estimates for $C_{m}\left[\Delta_{k}(x)\right](k, m \geqslant$ $2)$ could be clearly obtained by this method, on using the estimates for power moments of $|\zeta(\sigma+i t)|$. However, as transpires from [5, Chapter 8] and [20], such estimates have a rather complicated shape, and therefore a general form of the estimate for $C_{m}\left[\Delta_{k}(x)\right]$ would have an unwieldy exponent.

6. The number of nonisomorphic Abelian groups. We will consider now the problem of the distribution of nonisomorphic Abelian groups. As usual, let $a(n)$ denote the number of nonisomorphic Abelian groups with $n$ elements (see, e.g., [5, Section 14.5]). This is a multiplicative function and its generating series is

$$
\sum_{n=1}^{\infty} a(n) n^{-s}=\zeta(s) \zeta(2 s) \zeta(3 s) \cdots \quad(\sigma>1),
$$

so that many problems involving $a(n)$ are connected with power moments of $\zeta(s)$. If one sets

$$
\begin{gathered}
A(x):=\sum_{n \leqslant x} a(n)=\sum_{j=1}^{6} A_{j} x^{1 / j}+R_{0}(x), \\
A_{j}:=\prod_{k=1, k \neq j}^{\infty} \zeta\left(\frac{k}{j}\right),
\end{gathered}
$$

then $R_{0}(x)$ can be thought of as the error term in the asymptotic formula for the summatory function of $a(n)$. There is a wealth of results in the literature concerning the estimation of $R_{0}(x)$. In particular, the long-standing conjecture of H.-E. Richert that

$$
R_{0}(x) \ll_{\varepsilon} x^{1 / 4+\varepsilon}
$$

has been recently proved by Robert and Sargos [28]. In the other direction, Balasubramanian and Ramachandra [1] proved that

$$
R_{0}(x)=\Omega\left(x^{1 / 6}(\log x)^{1 / 2}\right),
$$

so that in fact the term $A_{6} x^{1 / 6}$ in (6.2) can be omitted in the definition of $R_{0}(x)(f=$ $\Omega(g)$ means that $\left.|f|=\Omega_{+}(g)\right)$. The author in [7] proved that

$$
\int_{1}^{X} R_{0}^{2}(x) d x=\Omega\left(X^{4 / 3} \log X\right),
$$

and in [6] he proved the upper bound

$$
\int_{1}^{X} R_{0}^{2}(x) d x \ll X^{39 / 29}
$$

Later, Heath-Brown in [3] improved this upper bound to be

$$
\int_{1}^{X} R_{0}^{2}(x) d x \ll X^{4 / 3}(\log X)^{89}
$$


which in view of (6.5) is the best possible up to a power of the logarithm. We will prove here the following theorem.

THEOREM 6.1. For any given $\varepsilon>0$,

$$
C\left[R_{0}(x)\right] \ll_{\varepsilon} x^{1 / 6+\varepsilon} .
$$

7. Proof of Theorem 6.1. For $\sigma>1$, we have, by using (6.2) and integration by parts,

$$
\begin{aligned}
\sum_{n=1}^{\infty} a(n) n^{-s} & =\zeta(s) \zeta(2 s) \zeta(3 s) \cdots \\
& =\int_{1-0}^{\infty} x^{-s} d A(x) \\
& =\int_{1}^{\infty} \sum_{j=1}^{6} \frac{1}{j} A_{j} x^{1 / j-1-s} d x+\int_{1-0}^{\infty} x^{-s} d R_{0}(x) \\
& =\sum_{j=0}^{6} \frac{A_{j}}{j s-1}+s \int_{1}^{\infty} R_{0}(x) x^{-s-1} d x
\end{aligned}
$$

with $A_{0}=R_{0}(1-0)$. By using the Cauchy-Schwarz inequality for integrals and (6.7), it follows that the last integral is absolutely convergent for $\sigma>1 / 6$. Therefore, the function

$$
\mathscr{A}(s):=\zeta(s) \zeta(2 s) \zeta(3 s) \cdots-\sum_{j=0}^{6} \frac{A_{j}}{j s-1}
$$

is regular for $\sigma>1 / 6$, and from (1.9) we have

$$
C\left[R_{0}(x)\right]=\frac{1}{2 \pi i} \int_{(1)} \mathscr{A}^{2}(s) s^{-2} x^{s} d s,
$$

similarly to (3.22). But we have, with $\sigma=1 / 6+\varepsilon$,

$$
\begin{aligned}
& \int_{T}^{2 T}|\mathscr{A}(\sigma+i t)|^{2} t^{-2} d t \ll T^{-2} \log ^{2} T \int_{T}^{2 T}\left|\prod_{j=1}^{5} \zeta(j \sigma+j i t)\right|^{2} d t+T^{-1} \\
& \ll T^{-1-10 \varepsilon} \log ^{2} T \int_{T}^{2 T}\left|\zeta\left(\frac{5}{6}-\varepsilon+i t\right)\right|^{2} \\
& \times\left|\zeta\left(\frac{2}{3}-2 \varepsilon+2 i t\right)\right|^{2}\left|\zeta\left(\frac{1}{2}+3 \varepsilon+3 i t\right)\right|^{2} \\
& \times\left|\zeta\left(\frac{2}{3}+4 \varepsilon+4 i t\right)\right|^{2}\left|\zeta\left(\frac{5}{6}+5 \varepsilon+5 i t\right)\right|^{2} d t+T^{-1},
\end{aligned}
$$

on using $\zeta(1+i t) \ll \log |t|$ and the functional equation for $\zeta(s)$. From the elementary inequality $|a b| \leqslant(1 / 2)|a|^{2}+(1 / 2)|b|^{2}$, it follows that the last integral does not exceed 
$\max \left(J_{T}, J_{T}^{\prime}\right)$, where

$$
\begin{aligned}
& J_{T}:=\int_{T}^{2 T}\left|\zeta\left(\frac{1}{2}+3 \varepsilon+3 i t\right)\right|^{2}\left|\zeta\left(\frac{2}{3}+4 \varepsilon+4 i t\right)\right|^{4}\left|\zeta\left(\frac{5}{6}-\varepsilon+i t\right)\right|^{4} d t \\
& J_{T}^{\prime}:=\int_{T}^{2 T}\left|\zeta\left(\frac{1}{2}+3 \varepsilon+3 i t\right)\right|^{2}\left|\zeta\left(\frac{2}{3}-2 \varepsilon+2 i t\right)\right|^{4}\left|\zeta\left(\frac{5}{6}+5 \varepsilon+5 i t\right)\right|^{4} d t .
\end{aligned}
$$

Following the proof of Heath-Brown's estimates for the analogous integrals in [3, equation (2.3)], we obtain that

$$
J_{T}+J_{T}^{\prime} \ll_{\varepsilon} T^{1+8 \varepsilon}
$$

This means that in (7.3) we may shift the line of integration to $\mathfrak{R e} s=1 / 6+\varepsilon$ and obtain

$$
\int_{(1 / 6+\varepsilon)} \mathscr{A}^{2}(s) s^{-2} x^{s} d s \ll_{\varepsilon} x^{1 / 6+\varepsilon},
$$

thereby proving Theorem 6.1.

8. The Rankin-Selberg convolution. We conclude our discussion by considering the error term connected to the so-called Rankin-Selberg series (see Rankin [26, 27])

$$
Z(s):=\zeta(2 s) \sum_{n=1}^{\infty}\left|a^{*}(n)\right|^{2} n^{1-\kappa-s}=\sum_{n=1}^{\infty} c_{n} n^{-s} \quad(\sigma>1),
$$

or the Rankin-Selberg convolution. Here, $a^{*}(n)$ denotes the $n$th Fourier coefficient of a holomorphic cusp form $\varphi(z)$ of weight $\kappa$ with respect to the full modular group $\mathrm{SL}(2, \mathbb{Z})$. We also suppose that $\varphi(z)$ is a normalized eigenfunction for the Hecke operators $T(n)$, so that $a^{*}(n)$ is a real-valued, multiplicative function. We have (see [8, 22]) $c_{n} \ll_{\varepsilon} n^{\varepsilon}$,

$$
\begin{gathered}
\sum_{n \leqslant x} c_{n}^{2} \ll_{\varepsilon} x(\log x)^{1+\varepsilon}, \\
\sum_{n \leqslant x} c_{n}=A x+\Delta(x, \varphi) \quad(A>0)
\end{gathered}
$$

with Rankin's classical estimate (see [26])

$$
\Delta(x, \varphi) \ll x^{3 / 5}
$$

We also have the mean-square estimate

$$
\int_{1}^{X} \Delta^{2}(x, \varphi) d x \ll_{\varepsilon} X^{2+\varepsilon}
$$

Rankin's estimate (8.4) withstands improvement for more than 60 years. The author gave heuristic reasoning in [8] for his conjectures that

$$
\Delta(x, \varphi) \ll_{\varepsilon} x^{3 / 8+\varepsilon}, \quad \Delta(x, \varphi)=\Omega\left(x^{3 / 8}\right),
$$


but these results at present are unattainable. Nevertheless, for $C[\Delta(\chi, \varphi)]$ we will prove a result with exponent better than $1 / 2$. This is the following theorem.

THEOREM 8.1. We have

$$
C[\Delta(x, \varphi)] \ll_{\varepsilon} x^{2 /(5-2 \mu(1 / 2))+\varepsilon},
$$

where

$$
\mu(\sigma)=\limsup _{t \rightarrow \infty} \frac{\log |\zeta(\sigma+i t)|}{\log t}
$$

COROLLARY 8.2. If the Lindelöf hypothesis that $\zeta(1 / 2+i t) \ll_{\varepsilon}|t|^{\varepsilon}$ holds, then

$$
C[\Delta(x, \varphi)] \ll_{\varepsilon} x^{2 / 5+\varepsilon} .
$$

COROLLARY 8.3. Unconditionally with $\mu(1 / 2) \leq 89 / 570$ (cf. [4]),

$$
C[\Delta(x, \varphi)] \ll_{\varepsilon} x^{285 / 668+\varepsilon}, \quad \frac{285}{668}=0.426646 \ldots
$$

9. Proof of Theorem 8.1. For $\sigma>1$, we have, on using (8.3),

$$
\begin{aligned}
Z(s) & =\int_{1-0}^{\infty} x^{-s} d\left(\sum_{n \leqslant x} c_{n}\right)=\int_{1-0}^{\infty} x^{-s}(A d x+d \Delta(x, \varphi)) \\
& =\frac{A}{s-1}+A+s \int_{1}^{\infty} \Delta(x, \varphi) x^{-s-1} d x
\end{aligned}
$$

since $\Delta(1-0, \varphi)=-A$. From (9.1), (8.5), and the Cauchy-Schwarz inequality for integrals, it follows that the last integral is absolutely convergent for $\sigma>1 / 2$. Therefore, similarly to (3.22), we obtain

$$
C[\Delta(x, \varphi)]=\frac{1}{2 \pi i} \int_{(1)} \frac{U^{2}(s)}{s^{2}} x^{s} d s
$$

where the function

$$
U(s):=Z(s)-\frac{A}{s-1}-A
$$

is regular for $\sigma>1 / 2$. The function $Z(s)$ satisfies the functional equation

$$
Z(s)=\psi(s) Z(1-s)
$$

with

$$
\psi(s)=(2 \pi)^{4 s-2} \frac{\Gamma(\kappa-s) \Gamma(1-s)}{\Gamma(s+\kappa-1) \Gamma(s)} \asymp|t|^{2-4 \sigma} .
$$


Thus, for $1 / 4 \leqslant \sigma \leqslant 3 / 4$, we obtain

$$
\int_{T}^{2 T}|Z(\sigma+i t)|^{2} d t \ll T^{4-8 \sigma} \int_{T}^{2 T}|Z(1-\sigma+i t)|^{2} d t
$$

This means that the problem is reduced to a bound for mean values of $|Z(\sigma+i t)|$.

For fixed $\sigma$ satisfying $1 / 2 \leqslant \sigma \leqslant 1$, we have

$$
\int_{1}^{T}|Z(\sigma+i t)|^{2} d t=T \sum_{n=1}^{\infty} c_{n}^{2} n^{-2 \sigma}+O_{\varepsilon}\left(T^{4-4 \sigma+\varepsilon}\right),
$$

proved by the author in [16]. The asymptotic formula (9.7) improves, for $3 / 4<\sigma \leq 1$, the result of Matsumoto [22] who proved

$$
\int_{1}^{T}|Z(\sigma+i t)|^{2} d t=T \sum_{n=1}^{\infty} c_{n}^{2} n^{-2 \sigma}+R(\sigma, T)
$$

with

$$
R(\sigma, T) \ll_{\varepsilon} \begin{cases}T^{5 / 2-2 \sigma+\varepsilon} & \left(\frac{3}{4}<\sigma<\frac{12+\sqrt{19}}{20}=0.81666 \ldots\right), \\ T^{60(1-\sigma) /(29-20 \sigma)+\varepsilon} & \left(\frac{12+\sqrt{19}}{20}<\sigma<1\right) .\end{cases}
$$

For $1 / 2<\sigma \leq 3 / 4$, our result is slightly weaker than the corresponding result of [22], namely,

$$
R(\sigma, T) \ll_{\varepsilon} T^{4-4 \sigma}(\log T)^{1+\varepsilon},
$$

but it should be remarked that (9.7) is a true asymptotic formula only in the range $3 / 4<\sigma \leq 1$. Recently, Sankaranarayanan [29] obtained new results on mean values of $|Z(\sigma+i t)|$. In particular, he proved that

$$
\int_{T}^{2 T}|Z(\sigma+i t)|^{2} d t \ll_{\varepsilon} T^{1 / 2+(4 \mu(1 / 2)+2)(1-\sigma)+\varepsilon} \quad\left(\frac{1}{2} \leqslant \sigma \leqslant \frac{3}{4}\right) .
$$

His result in [29], which for $3 / 4<\sigma \leq 1$ complements (9.11), is weaker than (9.7).

We can use (9.11) in conjunction with (9.6) to obtain

$$
\int_{T}^{2 T}|Z(\sigma+i t)|^{2} d t \ll_{\varepsilon} T^{4-8 \sigma+1 / 2+(4 \mu(1 / 2)+2) \sigma+\varepsilon} \quad\left(\frac{1}{4} \leqslant \sigma \leqslant \frac{1}{2}\right) .
$$

The exponent of $T$ on the right-hand side is less than two for

$$
\sigma>\frac{5}{12-8 \mu(1 / 2)}
$$

Hence by the method of the proof of Theorem 1.2, it follows that

$$
\int_{(c)} \frac{U^{2}(s)}{s^{2}} x^{s} d s \ll x^{c}
$$


with $c=5 /(12-8 \mu(1 / 2))$, and $1 / 2<1-c<3 / 4$ holds. This gives the bound

$$
C[\Delta(x, \varphi)] \ll_{\varepsilon} x^{5 /(12-8 \mu(1 / 2))+\varepsilon,}
$$

which is weaker than (8.7). If the Lindelöf hypothesis holds, then (9.15) gives

$$
C[\Delta(x, \varphi)] \ll_{\varepsilon} x^{5 / 12+\varepsilon} .
$$

From (9.15), we have unconditionally, with $\mu(1 / 2) \leq 89 / 570$,

$$
C[\Delta(x, \varphi)] \ll_{\varepsilon} x^{1425 / 3064+\varepsilon}, \quad \frac{1425}{3064}=0.46507832 \ldots
$$

To obtain the sharper bound in (8.7), we need a new mean value result for $F(s)$. This follows if we note that $F(s)$ factors as

$$
Z(s)=\zeta(s) D(s+\kappa-1)=\zeta(s) D_{0}(s)
$$

say, where $D_{0}(s)$ is a Dirichlet series which satisfies the functional equation

$$
D_{0}(s)=X(s) D_{0}(1-s)
$$

with

$$
\begin{aligned}
X(s) & :=\pi^{3 s-3 / 2} \frac{\Gamma((1 / 2)(\kappa-s)) \Gamma((1 / 2)(\kappa+1-s)) \Gamma((1 / 2)(2-s))}{\Gamma((1 / 2)(s+\kappa-1)) \Gamma((1 / 2)(s+\kappa)) \Gamma((1 / 2)(s+1))} \\
& \asymp|t|^{3(1 / 2-\sigma)}(|t| \geqslant 2) .
\end{aligned}
$$

This observation, made by Sankaranarayanan in [29], was crucial in proving the bound (9.11). Note that we also have

$$
D_{0}(s)=\sum_{n=1}^{\infty} d_{n} n^{-s}, \quad d_{n} \ll_{\varepsilon} n^{\varepsilon}(\sigma>1) .
$$

Thus the Dirichlet series $D_{0}(s)$ is quite similar to $\zeta^{3}(s)$, which satisfies the same type of functional equation as (9.19)-(9.20), namely,

$$
\zeta^{3}(s)=\chi^{3}(s) \zeta^{3}(1-s)
$$

with

$$
\chi^{3}(s)=8^{s} \pi^{3 s-3} \sin ^{3}\left(\frac{1}{2} \pi s\right) \Gamma^{3}(1-s) \asymp|t|^{3(1 / 2-\sigma)} \quad(|t| \geqslant 2)
$$

and the analogue of (9.21), which is

$$
\zeta^{3}(s)=\sum_{n=1}^{\infty} d_{3}(n) n^{-s}, \quad d_{3}(n) \ll_{\varepsilon} n^{\varepsilon}(\sigma>1) .
$$


This means that all properties of $\zeta^{3}(s)$, derived solely by the use of the functional equation (9.22)-(9.23) and the series representation (9.24), carry over to $D_{0}(s)$. In particular, the asymptotic formula

$$
\int_{1}^{T}|\zeta(\sigma+i t)|^{6} d t=T \sum_{n=1}^{\infty} d_{3}^{2}(n) n^{-2 \sigma}+O_{\varepsilon}\left(T^{3(1-\sigma)+\varepsilon}\right) \quad\left(\frac{1}{2} \leqslant \sigma \leqslant 1\right),
$$

which is [16, Corollary 1], was derived in this manner, using $\beta_{3}=1 / 3$ (cf. (4.4)), which follows from the functional equation (9.22)-(9.23) (see, e.g., [5, Chapters 3 and 13]). Therefore, we will obtain, as the analogue of (9.25), the asymptotic formula

$$
\int_{1}^{T}\left|D_{0}(\sigma+i t)\right|^{2} d t=T \sum_{n=1}^{\infty} d_{n}^{2} n^{-2 \sigma}+O_{\varepsilon}\left(T^{3(1-\sigma)+\varepsilon}\right) \quad\left(\frac{1}{2} \leqslant \sigma \leqslant 1\right) .
$$

Like (9.25), (9.26) is a true asymptotic formula only when $\sigma>2 / 3$. Using (9.26) in conjunction with (9.6), it follows that

$$
\int_{0}^{T}|F(\sigma+i t)|^{2} d t \ll_{\varepsilon} T^{2 \mu(1 / 2)(1-\sigma)+\varepsilon}\left(T+T^{3-3 \sigma}\right) \quad\left(\frac{1}{2} \leqslant \sigma \leqslant 1\right),
$$

which improves (9.11) for $1 / 2<\sigma \leqslant 3 / 4$ (for $\sigma=1 / 2$, the bounds are equal, although [29] contains the bound without $\varepsilon$, but with a log-power). Here, we used the bound $\mu(\sigma) \leqslant 2 \mu(1 / 2)(1-\sigma)$, which comes from convexity (since trivially, $\mu(1)=0$ ), but it should be remarked that, for some values of $\sigma$ in the range $1 / 2<\sigma<1$, better estimates than those furnished by convexity are known (see, e.g., [30, Chapter 5]).

To complete the proof, note that

$$
4-8 \sigma+2 \mu\left(\frac{1}{2}\right) \sigma+3 \sigma<2
$$

for

$$
\sigma>\frac{2}{5-2 \mu(1 / 2)}
$$

Then, similarly as before, we obtain

$$
\int_{(c)} \frac{U^{2}(s)}{s^{2}} x^{s} d s \ll x^{c}
$$

with $c=2 /(5-2 \mu(1 / 2))$, and $1 / 2<1-c<3 / 4$. This ends the proof of Theorem 8.1.

\section{REFERENCES}

[1] R. Balasubramanian and K. Ramachandra, Some problems of analytic number theory. III, Hardy-Ramanujan J. 4 (1981), 13-40.

[2] J. L. Hafner and A. Ivić, On the mean-square of the Riemann zeta-function on the critical line, J. Number Theory 32 (1989), no. 2, 151-191.

[3] D. R. Heath-Brown, The number of abelian groups of order at most $x$, Astérisque (1991), no. 198-200, 153-163. 
[4] M. N. Huxley, Integer points, exponential sums and the Riemann zeta function, Number Theory for the Millennium, II (Urbana, Ill, 2000), A K Peters, Massachusetts, 2002, pp. 275-290.

[5] A. Ivić, The Riemann Zeta-Function, A Wiley-Interscience Publication, John Wiley \& Sons, New York, 1985.

[6] — - The number of finite nonisomorphic abelian groups in mean square, HardyRamanujan J. 9 (1986), 17-23.

[7] _ The general divisor problem, J. Number Theory 27 (1987), no. 1, 73-91.

[8] _ Large values of certain number-theoretic error terms, Acta Arith. 56 (1990), no. 2, 135-159.

[9]___ Lectures on Mean Values of the Riemann Zeta Function, Tata Institute of Fundamental Research Lectures on Mathematics and Physics, vol. 82, Springer-Verlag, Berlin, 1991.

[10] - On the Laurent coefficients of certain Dirichlet series, Publ. Inst. Math. (Beograd) (N.S.) 53(67) (1993), 23-36.

[11] _ On the fourth moment of the Riemann zeta-function, Publ. Inst. Math. (Beograd) (N.S.) 57(71) (1995), 101-110.

[12] _ The Mellin transform and the Riemann zeta-function, Proc. Conf. on Elementary and Analytic Number Theory (Vienna, July 18-20, 1996) (W. G. Nowak and J. Schoißengeier, eds.), Universität Wien \& Universität für Bodenkultur, Vienna, 1996, pp. 112-127.

[13] - On the error term for the fourth moment of the Riemann zeta-function, J. London Math. Soc. (2) 60 (1999), no. 1, 21-32.

[14] On the integral of the error term in the fourth moment of the Riemann zeta-function, Funct. Approx. Comment. Math. 28 (2000), 105-116.

[15] _ On some conjectures and results for the Riemann zeta-function and Hecke series, Acta Arith. 99 (2001), no. 2, 115-145.

[16] _ On mean values of some zeta-functions in the critical strip, J. Théor. Nombres Bordeaux 15 (2003), 151-166.

[17] A. Ivić, M. Jutila, and Y. Motohashi, The Mellin transform of powers of the zeta-function, Acta Arith. 95 (2000), no. 4, 305-342.

[18] A. Ivić and Y. Motohashi, The mean square of the error term for the fourth power moment of the zeta-function, Proc. London Math. Soc. (3) 69 (1994), no. 2, 309-329.

[19]_ On the fourth power moment of the Riemann zeta-function, J. Number Theory 51 (1995), no. 1, 16-45.

[20] A. Ivić and M. Ouellet, Some new estimates in the Dirichlet divisor problem, Acta Arith. 52 (1989), no. 3, 241-253.

[21] M. Jutila, The Mellin transform of the square of Riemann's zeta-function, Period. Math. Hungar. 42 (2001), no. 1-2, 179-190.

[22] K. Matsumoto, The mean values and the universality of Rankin-Selberg L-functions, Number Theory (Turku, 1999) (M. Jutila and T. Metsänkylä, eds.), de Gruyter, Berlin, 2001, pp. 201-221.

[23] Y. Motohashi, An explicit formula for the fourth power mean of the Riemann zeta-function, Acta Math. 170 (1993), no. 2, 181-220.

[24]_ A relation between the Riemann zeta-function and the hyperbolic Laplacian, Ann. Scuola Norm. Sup. Pisa Cl. Sci. (4) 22 (1995), no. 2, 299-313.

[25] _ Spectral Theory of the Riemann Zeta-Function, Cambridge Tracts in Mathematics, vol. 127, Cambridge University Press, Cambridge, 1997.

[26] R. A. Rankin, Contributions to the theory of Ramanujan's function $\tau(n)$ and similar arithmetical functions. I. The zeros of the function $\sum_{n=1}^{\infty} \tau(n) / n^{s}$ on the line $\mathrm{R} s=13 / 2$. II. The order of the Fourier coefficients of integral modular forms, Math. Proc. Cambridge Philos. Soc. 35 (1939), 351-372. 
[27] R. A. Rankin (ed.), Modular Forms, Ellis Horwood Series in Mathematics and Its Applications: Statistics and Operational Research, Ellis Horwood, Chichester, 1984.

[28] O. Robert and P. Sargos, Three-dimensional exponential sums with monomials, to appear in J. reine angew. Math.

[29] A. Sankaranarayanan, Fundamental properties of symmetric square L-functions. I, Illinois J. Math. 46 (2002), no. 1, 23-43.

[30] E. C. Titchmarsh, Introduction to the Theory of Fourier Integrals, Oxford University Press, Oxford, 1948.

[31] W. P. Zhang, On the divisor problem, Kexue Tongbao (English Ed.) 33 (1988), no. 17, 14841485.

Aleksandar Ivić: Katedra Matematike RGF-a, Universiteta u Beogradu, Đušina 7, 11000 Beograd, Serbia and Montenegro

E-mail address: aivic@rgf.bg.ac.yu; eivica@ubbg.etf.bg.ac.yu 


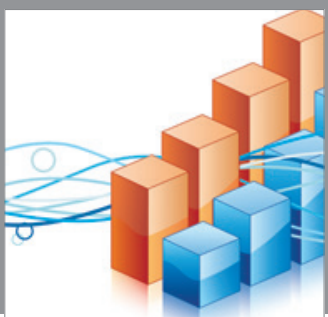

Advances in

Operations Research

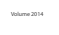

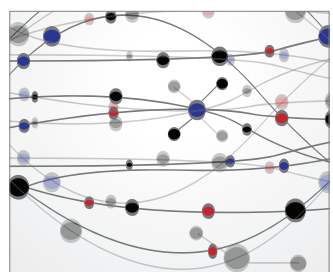

\section{The Scientific} World Journal
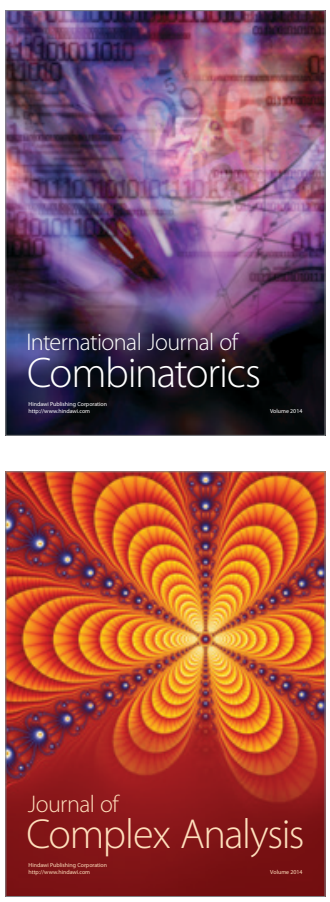

International Journal of

Mathematics and

Mathematical

Sciences


Journal of

Applied Mathematics
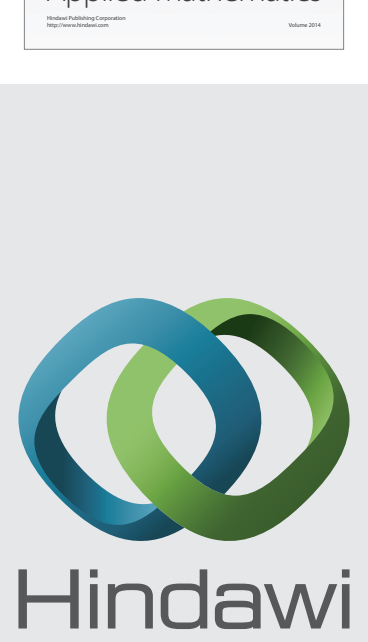

Submit your manuscripts at http://www.hindawi.com


Mathematical Problems in Engineering


Journal of

Function Spaces
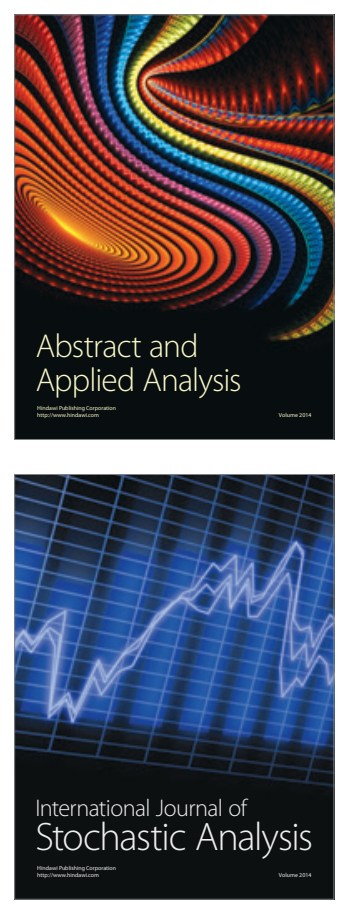

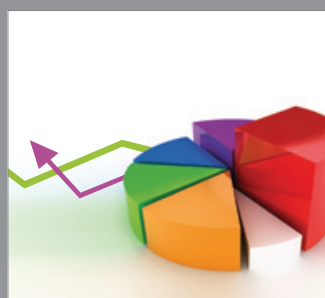

ournal of

Probability and Statistics

Promensencen
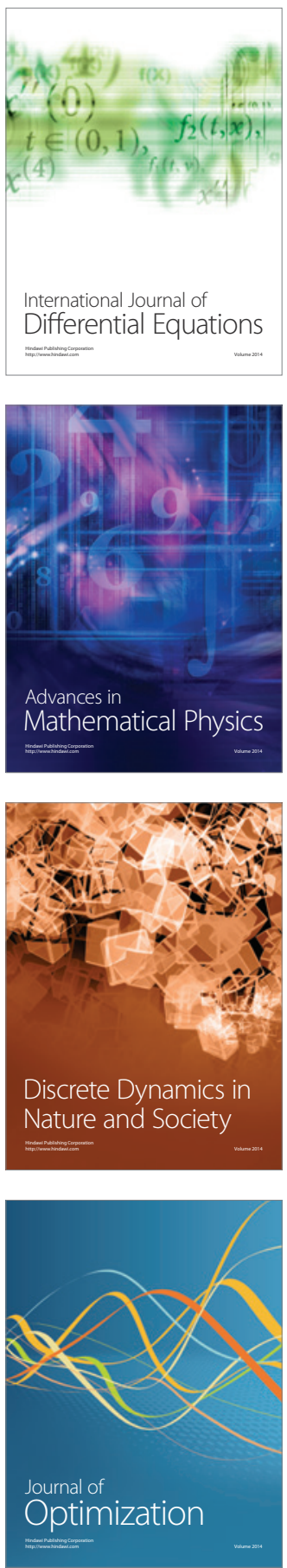